\title{
Treatment Decision-making for Early Prostate Cancer Patients - What Can Nurses Do
}

\author{
Jamie Irvine ${ }^{1}$, Siu Fung Chung ${ }^{1,2, *}$ \\ ${ }^{1}$ School of Health, The University of New England, Australia, Armidale, NSW, Australia \\ ${ }^{2}$ Kiang Wu Nursing College of Macau, MacauSAR, PRChina \\ *Corresponding Author: sfchung@kwmc.edu.mo
}

Copyright (C) 2014 Horizon Research Publishing All rights reserved.

\begin{abstract}
This review investigates the topic of treatment decision-making for early prostate cancer patients. The research question to be addressed is "What can oncology nurses do to support men with early prostate cancer in making treatment decisions?" Reports of primary research found by CIHNAL and Ovid search engines using the term "prostate cancer decision-making" are reviewed. A total of 18 studies have been identified. It is found that men with prostate cancer all need support and information when making treatment decisions. However, little has been said about the types of support and information needed or the best way to deliver them. The undesirable situation is coupled with people's seemingly innate wish to solve crises quickly and making decisions based on individual experience and misconception. As such, reciting treatment options simply and handing out generic literature cannot ensure that the patient and his family members fully understand the options available. It is argued that individualized treatment, with informed decision made jointly by the patient, his family and a treating team, offers the best chance for satisfactory outcomes. Nurses can make use of their research to design interventions and inform the patient of the treatment approach to better assist men in making treatment decisions. It is hoped that individualized and patient-centered treatment will become the norm in healthcare.
\end{abstract}

Keywords Early Prostate Cancer Patients, Treatment Decision Making, Support of Oncology Nurses

\section{Introduction}

This review investigates the topic of treatment decision-making for early prostate cancer patients. The research question this review addresses is "What can nurses do to support men with early prostate cancer in making treatment decisions?"

The question arose when the topic "the experience of men making decisions about treatment for early prostate cancer" was considered. A research problem was then identified in this topic, which concerns the trouble that men may encounter in considering treatment options for early prostate cancer, given that there are several choices available but none has been scientifically proven and favourable $[1,2]$. Long disease-free periods leave many men to wrestle with quality-of-life issues and the perception that they have cancer in their bodies. Men with localized prostate cancer are at risk of decisional regret if they feel they have made the wrong decision, especially for younger men who may have to live with urinary and sexual dysfunction for long periods of time [3]. Some reviewers put decisional regret in prostate cancer patients as being between 4-19\% [4]. This paper looks at how prostate cancer patients prefer to make decisions on their treatment, what information they require, what media are appropriate, when the best time is for nurses to offer information and how long the support from nurses should last.

\section{Scope of the Problem and Definition of Key Terms}

Treatment decisions for men with early stage prostate cancer are complicated by a lack of scientific consensus on the most effective treatment options available $[1,4]$. This is compounded by the growing number of men diagnosed with early prostate cancer at younger ages due to the wider availability of screening [5]. Prostate specific antigen (PSA) tests can show rises of an indicator of prostate hypertrophy in blood, but there are other reasons for PSA to increase, except for the presence of malignancy. For example, benign prostatic hypertrophy or infection may cause higher PSA levels [1]. There are currently debates on who should have prostate cancer screening and when should it be done. A large body of literature on decision-making for screening exists but this topic falls beyond the scope of this paper.

Many cancers detected solely by PSA tests are low-risk cancers, having little or no impact on life expectancy [6]. Data from the United States suggests that although one sixth of men in the U.S. will be diagnosed with prostate cancer, only one in 35 will die from it. Biopsies can only reveal if a 
mass is prostate cancer; they do not tell how fast the mass may grow [6]. Graham et al [6] argue that one of the big challenges in prostate cancer is to decide which men have rapidly growing tumours and which have slow growing ones that will never bother them. This can lead to confusion over which treatment option men with early prostate cancer should choose, as the side effects of treatment including incontinence, impotence and bowel complications can be profound. This is again compounded by the fact that men being diagnosed at younger ages will have to live with these complications for longer periods of time [5].

The U.K. based National Institute for Health and Clinical Excellence (NICE) has made recommendations for treatment of early, localised prostate cancer based on systematic views of evidence. According to its definition, men with a PSA level of $<10 \mathrm{ng} / \mathrm{ml}$, a Gleason score of $<6$ and a tumour clinical stage of T1-T2 are at low risk. NICE recommends active surveillance, monitoring PSA levels and repeating biopsies as the best treatment option for these men [6]. A 2009 report by the U.S. Agency for Healthcare Research and Quality finds that no one treatment for localized prostate cancer can be considered preferred and that individual patients must decide on treatments based on their own tradeoffs between treatment effectiveness, necessity and side effects [7]. Neither do Australia's National Health and Medical Research Council's (NHMRC) 2002 clinical practice guidelines for managing localized prostate cancer give any clear recommendation on prostate cancer treatment due to the lack of evidence for superior choice [8]. The Council does, however, advise that a man's life expectancy, health status and tumour-related factors be taken into consideration when deciding on treatment and that all men diagnosed with localized prostate cancer should at least have the treatment options of prostectomy, radiotherapy and watchful waiting explained to them [8].

Decision-making affects a patient's quality of life (QOL) by impacting the physical, social, psychological and spiritual aspects of a person's life. People making decisions report disruptions to their QOL, including sleep disruption, over-eating, headaches and loss of appetite [1]. Men with early stage prostate cancer have additional stress in decision making because of the lack of scientific consensus on the best treatment options.

Over the past few years, literature on treatment decision-making for prostate cancer has focused on informed decision-making and shared decision-making, which are regarded as the golden standard for treatment decisions [9]. In informed decision-making, patients make their decision when they understand their disease, the risks and benefits of treatment options and have weighed these against their own values and outcome desires. Shared decision-making occurs when a physician collaborates with the patient to make a mutually satisfactory treatment decision[9]. This process of understanding is central to the notion of informed consent, which is a necessary step in any treatment.

In this review, what oncology nurses can do to assist men to make treatment decisions will be examined. Nurses are involved in patient care from the moment of diagnosis and they are often central to orientating the patients to the "cancer world" of modern oncology treatment. Making appointments for potentially several rounds of treatment extending over months, educating patients about how the treatments work and their side effects, as well as providing the patients with ongoing physical and psychosocial care for them to move through the cancer experience, are different parts of the nurse's role. For prostate cancer patients, the time of decision-making is the most emotionally fraught [10]. Moreover, patients are now expected to participate in decision-making. Through researches, nurses will acquire the knowledge about what information can be best delivered through which media and when is the best time to approach patients to offer education and information in order to help patients reach individual decisions on their treatment.

\section{Literature Sources Accessed for This Review}

The reports discussed in this review are primary source reports on research. They were found by CIHNAL and Ovid search engines using the term "prostate cancer decision-making". The * was also used in CIHNAL searches to truncate the records. Searches were limited by date "2000-current" to preserve relevance. Access to reports was further limited by the access of the search engines to full text or PDF articles. The reports included in the review are primary source reports on researches into the treatment decision-making phase of people with localized prostate cancer. Reports on decision-making by men with local and advanced prostate cancer have also been included. Once a report had been found, a search would be done on its reference list to look for citations on other research reports and a descendancy search would be conducted for research reports in which the article has been cited $[2,11]$. Reports excluded from the review were secondary source research documents, although they were used in gaining an overview of the topic, and primary research reports dealing with decision-making about screening for prostate cancer.

In all, 18 research reports match the criteria and are available for the review. Two of the reports are reports on randomised clinical trials of intervention tools $[12,13]$. One is a qualitative and quantitative assessment of a decision-making aid [16]. Eight are reports on studies employing questionnaires [10,15-21]. One is a report of quantitative and qualitative analysis of an online discussion group [22]. One study is a secondary review of videotaped treatment consultations used as part of a longitudinal study [23] while two others report on pilot studies of treatment decision tools $[24,25]$. The other ones are a series of interviews [26], one consisting of a single in-depth interview [9] and one consisting of results from a quantitative survey [5]. The final study is a report on developing a health literacy scale [27]. The sample sizes of these reports range from 13 to 804 participants. The reports come from the United States, 
Canada, Germany, Holland and Australia.

\section{Results}

For the majority of men, the time following diagnosis is the most emotionally fraught period [10.13]. Men in one study spoke of being 'blindsided' by the diagnosis and described feelings of shock and anxiety [10]. Men who participated in evaluation of decision-making tools after their treatment stated that the information contained in the tools was very useful to them at the time of diagnosis [25]. Mishel et al. [12] assert that during this time, uncertainty generates fear and consequentially leads to fear-based decisions and a desire for rapid treatment. The consistent popularity of a surgical treatment option is a testament to this assertion. Surgery was the most frequently selected treatment across all studies. Half of the patients in the study of Sidana et al [5] chose surgery because they would not feel satisfied until the prostate and tumour were removed from their bodies. The men in the study of Ramsay et al. [20] considered surgery the definitive treatment. Those who used the decision-making tool and test in the study of Taylor et al.[13] were mainly interested in re-visiting treatment options which aimed at cure and most of such patients chose surgery. Denberg, Melhano and Steiner [9] conclude that the men in their study 'seemed as interested (and sometimes more so) in eliminating fear and uncertainty in the short term than in realizing cure over a longer term'. The researchers relate this to decision science, stating that an early, primitive reaction to any personally relevant object is a good-bad dichotomy. An antidote to this fear, according to the theory of Uncertainty in Illness, is information which can be processed by the patient and his support group and used to make informed treatment decisions [12]. The two most feared side effects across multiple studies were urinary and sexual dysfunction $[5,10,13]$.

The seeking of information is a major theme. Three quarters of the 132 men in the study of Taylor et al.[13] accessed the decision-making tool to gain more information. Out of the 493 men in the study of Sidana et al.[5] 59\% of them sought information from three or more sources. The average number of sources for information among the 804 men in the study of Ramsay et al.[20] was 4.9 items. Snow et al. [21] found that only $2 \%$ of their 138 Canadian men who responded to a questionnaire about their information needs after diagnosis did not want more information regarding localized prostate cancer. The researchers also found evidence of a knowledge gap with regard to knowledge of all treatment options, details of specific treatments, the risks and benefits associated with each treatment and personal considerations, such as recovery time after treatment $[4,21]$. Feldman-Stewart, Brennenstuhl, Brundage \& Seimanns [16] had similar findings. $90 \%$ of their subjects wanted more information on localized prostate cancer but the researchers highlighted that the types and amount of information these men wanted varied considerably. The team went on to demonstrate in a later study that all men with localized prostate cancer wanted different types of information, but it was impossible to predict the type of information any one man would want in order to feel fully informed [17]. Reichlin et al. [14] designed an interactive electronic game to assist men in making treatment decisions. The tool featured an option which allowed individual men to print questions pertaining to their treatment side-effects and bring these to their doctors for clarification. Ten of the 13 subjects had kept a journal or made notes during their information-gathering stage following their diagnosis and stated that they had printed the questions for their doctor [14]. Although the sample of 13 American men is small, it can be noted that the majority of the men were actively seeking information and wanted to make use of the questions generated by the computer.

The subjects in multiple studies report that they are overwhelmed by the amount of information and the contradictory nature of such information $[9,16,20]$. Many of the studies show that men access written and verbal information from books, pamphlets, government publications, physicians and other health professionals, family members, and other people who have had cancer. The Internet has become the most popular source of information after physicians and personal contacts $[5,13,20,22]$. The use of a CD ROM and two other electronic-based decision-making tools indicate that men are willing to access information across multiple media $[13,14,25]$. Although the use of electronic media and the internet offers a huge number of sources of information for men with prostate cancer, much of the information may be incorrect or biased [20,22].

Men's participation in the decision-making process was linked to their satisfaction after treatment. A study by Fischer et al. found that of the 126 Dutch men in the study, $78 \%$ felt they had either participated in shared decision-making $(60 \%)$ or made autonomous decisions (18\%). Men under 65 and men with localized prostate cancer made up the majority of these men. A total of $22 \%$ felt that they had no input into their decision. Of these men, none of the men under 65 years old were very satisfied with their treatment at six months, and two thirds were either quite dissatisfied or neither satisfied nor dissatisfied. The patients who felt they had participated least in decision-making in the study reported only having one treatment option offered to them [18]. In the study of Snow et al. with 138 subjects, those who had not been offered adequate information were more likely to experience anxiety or depression [21]. Although the hypothesis of Christie et al. in their study on 57 American men that more time discussing treatments would lead to better emotional adjustments at follow up was not confirmed, the study found that people who discussed treatment with their social group had a less negative effect at one and six months, while those who spent time talking more to their medical team had an increase in positive effect at one month and a trend towards a significant positive effect at six months [15]. Across multiple studies, varying proportions of men stated that they would prefer to have no part in their 
decision-making $[18,26,13]$.

In all of the research literature, the use of social support networks is a major theme. Men consistently seek information and emotional support from other people beyond the medical system. Christie et al. [15] found that men spend considerably more time discussing treatment options with their family members and friends than with a medical team. In the study of Sidana et al [5] $50 \%$ of the men discussed treatment options with their wives. Boehmer \& Babayan conducted a study of interviews with 21 men with localized prostate cancer and found that 18 of them trusted other people in their decision-making process. For these men, the trusted people were a source of emotional support and information. The female partners of 11 men attended the treatment decision consultation and seven female partners actively participated in it [24]. Most of the 17 men in Wallace \& Storm's study [10] stated that they reached a final treatment decision after discussing it with their wives. Discussing treatment options with non-medical sources was found to encourage men to look more deeply into them and also provided therapeutic emotional support in a German study, which analyzed 81 discussion threads in an online PC support group [22]. This research demonstrates that a large majority of men have support from people with whom they will discuss treatment options. It is important that health professionals identify these people and ensure that they too have access to relevant, accurate knowledge.

The way that men reach decisions is discussed in many reports. An Australian study by Steginga et al. looked into the decision-making processes of men diagnosed with localized prostate cancer. 108 men were interviewed about their thoughts around treatment decisions. Of these respondents, 91\% used 'non-systematic' decision-making processes. The researchers defined non-systematic decision-making process as deferring to the doctor, positive and negative recollections of others' cancer experience, and a pre-existing belief that surgery was the best option for cure. Of these men, $48 \%$ reported that they would defer to their doctor's decision while $47 \%$ said that when they made decisions, they considered the experience of their acquaints who had cancer. Some men in this group stated that they were distressed by their doctor's insistence that they should make a decision [26]. In a trial of a tool designed to assess the misconceptions about treatment choices in men with localized prostate cancer, Beydoun et al. [27] found that although all of their 184 participants with localized prostate cancer had already chosen a treatment option, most of them gave incorrect answers to questions relating to common complications, the possible duration of side effects, the safe timeframe (up to six months) within which decisions should be made, as well as the grade of their own cancer, which indicates its risk of spreading. Denberg, Melhado \& Steiner interviewed 20 men who had been diagnosed but not yet treated for localized prostate cancer on their knowledge of their disease. Although all patients had been taken through all available treatment options by their urologist, none of the patients explicitly compared treatments in regard to their relative likelihood of side effects. Some patients associated major side effects as being part of every treatment without regarding the different probabilities and severity among the treatments. Half of the patients dismissed side effects altogether, asserting that anything was better than dying. Nineteen patients used anecdotes from the cancer experiences of others when reviewing options and, the researchers assert, put more emphasis on this data than population based data. Sixteen of the men stated that they would not seek a second opinion due to a false belief that a second opinion is only used to confirm diagnosis (six men), and that it would increase uncertainty or delay treatment (10 men). The researchers assert that this study demonstrates that providing information alone is not consistent with a patient-centered model of care and that informed and shared decision-making models need to take into consideration the role that fear plays in desiring a rapid treatment, the role of influential popular misconceptions in decision-making and the propensity of people to make decisions based on anecdote[9]. These studies also highlight a role for nurses in coaching a patient through his diagnosis and treatment decision. Nurses can provide them with specific information, check that they understand the information using teach-back method, challenge misconceptions and anecdotes and help the patients generate questions for their physicians who will help them clarify their individual uncertainties.

A physician's recommendation is the most influential factor in the majority of men's decisions across multiple studies $[10,20,26]$. However, they are often given one treatment option only. Of the 493 men studied by Sidana et al. [5], $81 \%$ were recommended only one treatment option. In the study of Snow et al [21] half of the men who visited an urologist were not given radiotherapy as an option. Taylor et al. [13] suggest that the increased satisfaction with the decisional tool assessed in their study reported by men who desired less control and accessed fewer other sources of information indicates that clinicians need to provide information to patients who are not actively seeking it. Clinicians should be included to actively invite patients in shared decision-making. The shared decision-making (SDM) skills of radiation oncologists was studied by Pieterse et al. In this study SDM skills were defined as presenting options, discussing benefits and harms of treatment, clarifying patients concerns, clarifying understanding, making decisions and providing follow-up. The researchers measured the physicians' SDM skills in response to 25 patients' appraisals, which they define as any utterance conveying strength of preference, personal importance, positive or negative evaluation, attitude outcome expectation or weighing of a treatment aspect or option. The study found that the essential SDM skills were inconsistent. More often than not, the potential harms of a treatment may be included rather than the benefits of different treatments. The radiation oncologists in the study also only sought patients' preferences or needs for questions in the later stages of the treatment discussion when there was not a lot of time to properly clarify a patient's understanding. The study also 
found that while a minority of radiation oncologists explicitly or implicitly requested patient appraisals, some patients only offered appraisals when the radiation oncologists sought them. In half of the cases, the oncologists amplified the patients' appraisals by allowing them to further explore patient values and preferences and to clear up any misconceptions. Overall, the study found that the radiation oncologists did not generally seek patient preference during treatment discussions but that when they sought a patient's opinion, it allowed a more thorough exploration of treatment options [23]. Across the studies, demographic information alone is not enough to ascertain what information a patient may need $[13,20,22]$. This points to an argument that preferences about treatment options should be actively sought from patients in order to better deliver effective and individualized treatment options [23]. The data highlight the need for patients to be invited, or even encouraged to ask questions and discuss their treatment. Again, there is a role for nurses to assess patient's personal understanding of treatment, provide individual information and clarify what other information or support they need to make a decision.

\section{Discussion and Focus}

The data outlined above show that men with localized prostate cancer all require support and information when making treatment decisions. Demographic studies reveal that there is little heterogeneity of what type of support and information is needed or what is the best way to deliver them. Coupled with people's seemingly innate need to solve crises quickly and to make decisions based on individual experience and misconception, this has created a situation in which simply reciting treatment options and handing out generic literature cannot ensure that a patient and their family fully understand their options. A complex and time-poor medical system that is increasingly more difficult to explain and understand adds to the dilemma. From the literature, it is plain that individualized treatments involving shared decision-making between a patient, their family and a treating team offer the best chance to ensure informed decision-making, which may give the most satisfactory outcomes for the patient. The study of Mischel et al. [12] best illustrates what types of intervention may best deliver these outcomes. This study will be covered in detail as we believe it points to an answer to the research question most effectively in that it offers a nurse-driven practical intervention to assist men in making decisions at the time they need the support to do so. These researchers developed an intervention for men to use prior to visiting their physician to have a treatment discussion. The intervention was designed to assist men in learning more about prostate cancer, its treatments, their side effects, as well as how best to provide the physician with information about the patient's medical and psychosocial history, how to ask questions, clarify information, assess information and assert his treatment preference. The intervention consisted of a booklet, a DVD and four nurse follow-up phone calls. The booklet included a tear out sheet, which helped men identify their individual concerns, and prompted them to ask questions of the doctor. The nurse phone calls then helped the patient clarify his understanding and refine questions for the physician. The sample size was 256 American men with localized prostate cancer randomly assigned to one of three groups: a control group, a treatment direct (TD) group in which only the patient received the information package, and a treatment supplemented (TS) group in which the patient and a primary support person were given the information. Measurements of uncertainty were taken at recruitment, immediately following diagnosis, at four weeks following the treatment decision discussion and at three months post-entry, after patients had commenced treatment. The study found an improvement in the intervention groups over the control group at the second measurement in cancer knowledge and problem-solving. Members of this group were also given more information by their physicians at the treatment decision discussion, shared more information with their physician and participated in the decision-making process more. The improvement in cancer knowledge and problem-solving had diminished at the third measurement. At the third measurement, men in the intervention group had less decisional regret, less anxiety and depression, a higher quality of life and continued to seek information more often and from a wider variety of sources than men in the control group [12]. This is important information as by the third measurement, side effects of treatment had begun to affect men in the study. It suggests that the intervention not only assisted men in making treatment decisions but also empowered them in managing side effects and participating in their own health care by being informed, asking relevant questions and seeking and finding information. This intervention is noteworthy as it offers men a tool to improve their knowledge and health communication and problem-solving skills. It is delivered at the right time, during the uncertain period immediately following diagnosis, and delivered through a variety of methods including written material, electronic material and personal phone calls. It also individualizes care, includes support people and educates patients on how to manage their health in the long term.

Again, the research highlights the nurse as an educator and coach. During the follow-up phone calls, the nurse would ask the patient about what areas of concern they had, provide information about their concerns and help the patient to formulate relevant, pertinent questions to ask their physician during their treatment discussion. Nurses could also assess men's personal preferences and social supports to further individualize treatment. This process both improves the patient's health literacy and better enables them to participate in their treatment decision by ensuring that they understand their own situation and options under the increasingly technology-based, jargon-filled and institutionalized health system.

\section{Conclusion}


Men with a diagnosis of localised prostate cancer must choose between a number of treatment options, most commonly watchful waiting, radical prostatectomy, brachytherapy and external beam radiotherapy. A challenge for these men is the fact that no single option has been shown to be most effective for treating prostate cancer. These men must consider their quality of life and the side effects of each treatment during a time of high emotional stress. Providing these men with accurate, assessable and comprehensible information is part of the support they need for making their decisions. Men must be encouraged to consider their own personal preferences for treatment and people who can give them support must be included in that process. Nurses can better assist men in making treatment decisions by using researches to design interventions and informing them of the treatment approach. Prevalence of this practice will ensure that individualized, patient-centered treatment becomes the norm in healthcare.

\section{REFERENCES}

[1] ORourke, M. 2007, Choose wisely: therapeutic decisions and quality of life with prostate cancer. Clinical Journal of Oncology Nursing. 11, 40 1-08.

[2] Polit, D.F. \& Beck, C. T. (2012) Nursing Research: Generating and Assessing Evidence for Nursing Practice. Philadelphia. Lippincott Williams \& Wilkins.

[3] Cox, J. \& Amling, C.L. (2008).Current decision-making in prostate cancer. Current Opinion in Urology. 18(3). 275-278.

[4] Birnie, K. \& Robinson, J. (2010).Helping patients with localized prostate cancer reach treatment decisions. Canadian Family Physician 56(2). 137-141.

[5] Sidana, A., Hernandez, D.J., Feng, Z., Partin, A.W., Trock, B.J, Saha, S. \& Epstein, J.I. (2012).Treatment decision making for localized prostate cancer: what younger men choose and why. The Prostate. 72, 58-64.

[6] Graham, J., Baker, M., Macbeth, F. \& Titshall, V. (2008).Diagnosis and treatment of prostate cancer: summary of NICE guidance. British Medical Journal. 366, 610- 613.

[7] Kuban, D. (2009).Localised prostate cancer: The battle of treatment options enters the larger arena. Oncology, 23(10), $867,873$.

[8] National Health and Medical Research Council (2002). Clinical Practice Guidelines: Evidence-Based Information and Recommendations for the Management of Localised Prostate Cancer. Commonwealth of Australia. Retrieved at: http files_nhmrc/publications/attachments/cp88.pdf

[9] Denberg, T. D., Melhado, T. V. and Steiner, J. F. (2006), Patient treatment preferences in localized prostate carcinoma: The influence of emotion, misconception, and anecdote. Cancer, 107: 620-630.

[10] Wallace, M. \& Storms, 5. (2008).The needs of men with prostate cancer: results of a focus group study. Applied
Nursing Research 20 (4).

[11] Andrews, I., Sullivan, G., \& Minichello, V. (2004).The philosophical and theoretical context of qualitative research. In Minichiello, V., Sullivan, G., Greenwood, K. \& Axford, R. Research Methods for Nursing and Health Science (pp. 59-69). Frenches Forest, NSW, Prentice Hall.

[12] Mishel, M.H., Germino, B.B., Lin, L., Pruthi, R.S., Wallen, E.M., Crandell, J., Blyler, D. (2009). Managing uncertainty about treatment decision making in early stage prostate cancer: a randomised clinical trial. Patient Education and Counseling. 7 7(3), 349-3 59.

[13] Taylor, K.L., Davis, K.M., Lamond, T., Williams, R.M., Schwartch, M.D. \& Lawrence, W. (2010).Use and evaluation of a CD-ROM-based decision aid for prostate cancer treatment decisions. Behavioural Medicine. 36, 3 $0-140$.

[14] Reichlin, L., Nithya, M., McArthur, K., Harris, A.M., Rajan, N. \& Drasco, C.C. (2011).Assessing the acceptability and usability of an interactive serious game in aiding treatment decisions for patients with localised prostate cancer.Journal of Medical Internet Research. 13.

[15] Christie, K., Meyerowitz, B.E., Giedzinska-Simons, Gross, M. \& Agus, D.B. (2009).Predictors of affect following treatment decision-making for prostate cancer: conversations, cognititve processing, and coping. Psycho-Oncology. 18. 508-514.

[16] Feldman-Stewart, D., Brennenstuhl, S. \& Brundage, M.D. (2008). The information needed by Canadian early-stage prostate cancer patients for decision making: stable over a decade. Patient Education and Counselling. 17, 437-442.

[17] Feldman-Stewart, D., Brennenstuhl, S., Brundage, M.D. \& Siemens, D.R. (2009).Overall information needs of early stage rostate cancer patients over a decade: highly variable and remarkably stable. Support Care Cancer 17. 429-43 5.

[18] Fischer, M., Visser, A., Voerman, B., Garssen, van Andel, G., Bensing, J. (2006)Treatment decision making in prostate cancer: Patients participation in complex decisions. Patient Education and Counselling.63. 308-3 13.

[19] Stewart, D.F., Brennenstuhl, S. \& Brunbridge, M.D. (2008). The information needed by Canadian early stage prostate cancer for decision-making: Stable over a decade. Patient Education and Counseling.73 (3) 437-442.

[20] Ramsay, S.D., Zeliadt, S.B., Arora, N.K, Potosky, A.L., Blough, D.K., Hamilton, A.S., Van Den Eeden, S.K., Oakley-Girvan, I. \& Penson, D.F. (2009).Access to information sources and treatment considerations among men with local stage prostate cancer. Urology. 74, 509-515.

[21] Snow, S.L., Panton, R.L., Butler, L.J. Wilke, D.R., Rutledge, R.D.H., Bell, D.G. \& Rendon, R.A. (2007).Incomplete and inconsistent information provided to men making decisions for treatment of early-stage prostate cancer. Urology. 69(5).

[22] Huber, J., Ihrig, A. Peters, T., Huber, C.G., Kessler, A., Hadaschik, B., Pahernik, S. \& Hohenfellner, M. (2010).Decision-making in localized prostate cancer: lessons learned from an online support group. British Journal of Urology International. 107, 1570-1575.

[23] Pieterse, A.H., Henselmans, I., Hanneke, C.J.M., Koning, C.C.E., Geijsen, E.D. \& Smets, E.M.A. (2011), Shared 
decision making: Prostate cancer patients appraisal of treatment alternatives and oncologists eliciting and responding behaviour, an explorative study. Patient Education and Counselling. 85, 251-259.

[24] Boehmer, U. \& Babayan, R.K. (2005).A pilot study to determine support during the pre-treatment phase of early prostate cancer. Psycho-Oncology. 14. 442 -449.

[25] Berry, D.L. (2010). Development and evaluation of the personal patient profile- prostate (P3P), a web based decision support system for men newly diagnosed with localized prostate cancer. Journal of Medical Internet Research. Vol. 12 (4).
[26] Steginga, S.K., Occhipinti, S., Gardiner, R.A., Yaxley, J. \& Heathcote, p. (2002).Making decisions about treatment for localized prostate cancer. British Journal of Urology International. 89, 2 55-260.

[27] Beydoun, A.H., Mohan, R., Beydoun, M.A., Davis, J., Lance, R., Schellhammer, P. (2010). Development of a scale to assess patient misperceptions about treatment choices for localized prostate cancer. British Journal of Urology International. 106(3).334-341. 